\title{
Où en sommes-nous avec l'implication des parties prenantes?
}

\author{
Marie-Pier Marchand \\ Université du Québec à Montréal
}

Résumé: Cet article s'intéresse à la question de l'implication des parties prenantes à partir d'un survol de la littérature existante dans le domaine de l'évaluation. L'article permet de mieux définir les dimensions essentielles liées à cette thématique, notamment en abordant le concept de parties prenantes et en discutant des différentes approches favorisant leur implication, des diverses formes sous lesquelles apparait cette implication ainsi que des critères conduisant à leur sélection. Lauteure tente d'y mettre en exergue la valeur ajoutée du recours à une démarche évaluative impliquant les parties prenantes, tout en exposant les enjeux reliés à un tel exercice.

Mots clés : évaluation collaborative, évaluation participative, parties prenantes

\begin{abstract}
This paper focuses on stakeholders' involvement in program evaluation and provides an overview of the current literature on this topic. The paper aims to conceptualize stakeholders' involvement from the definition of key dimensions related to this theme, in particular by addressing the concept of stakeholders, discussing different approaches and various forms of stakeholders' involvement, and identifying criteria for selection. The author tries to highlight the added value of using an evaluation process involving stakeholders, while exposing the issues related to such an exercise.
\end{abstract}

Keywords: collaborative evaluation, participatory evaluation, stakeholders

\section{INTRODUCTION}

L'implication des parties prenantes, c'est-à-dire l'ensemble des personnes concernées ou interpellées à des degrés divers par l'évaluation d'un programme et ses résultats, demeure un enjeu fondamental à la pratique évaluative telle qu’observée de nos jours (Chen, 2004; Mark, Greene et Shaw, 2006; Orr, 2010; Schwandt, 2009). Bien quelle puisse entraîner son lot de tensions (Hurteau, 2009) et que tous n'y adhèrent pas sans réserve (Daigneault et Jacob, 2012), nombreux sont les praticiens et théoriciens qui prônent l'importance de la participation des parties prenantes au processus évaluatif de par les avantages quelle procure, d’où la naissance et l'effervescence des multiples approches inclusives.

Le présent article propose de revisiter la thématique de l'implication des parties prenantes en effectuant une synthèse des écrits relativement aux dimensions 
essentielles à considérer pour la pratique évaluative en lien avec cette thématique. La principale contribution des propos qui s'y retrouvent réside dans le fait qu'il existe à l'heure actuelle très peu de références en langue française traitant du sujet et se concentrant sur de telles orientations. En plus de permettre au lecteur de mieux conceptualiser l'implication des parties prenantes, cet article s'avère utile pour lévaluateur qui se questionne quant aux tenants et aboutissants d'une telle pratique en le guidant dans sa réflexion.

\section{DÉMARCHE ET OBJECTIFS}

Afin de mieux comprendre les diverses composantes inhérentes à l'implication des parties prenantes, l'auteure a entamé une démarche de type exploratoire, visant à recenser et à examiner les écrits traitant de cette thématique. Les propos qui suivent émergent des constats et des apprentissages effectués par l'auteure à la suite de ses lectures et analyses; ils exposent différents regroupements théoriques afin déclairer le concept à l'étude et proposent certains aspects à considérer dans la mise en œuvre d'une évaluation impliquant les parties prenantes.

\section{L'IMPLICATION DES PARTIES PRENANTES : LES CONNAISSANCES ACTUELLES}

D’emblée, il importe de préciser qu'un débat persiste dans la littérature à légard de la terminologie employée pour référer aux évaluations impliquant les parties prenantes : doit-on parler dévaluation participative ou dévaluation collaborative, la participation et la collaboration étant deux formes d'implication des parties prenantes poursuivant des visées distinctes? Tel que le suggèrent Fetterman, Rodriguez-Campos, Wandersman, et O'Sullivan (2013), afin déviter ce flou terminologique, nous recourrons dans le contexte actuel de cet article aux termes génériques et plus englobants dévaluations qui impliquent les parties prenantes, combinant du coup lensemble des approches qui favorisent cette implication, indépendamment de la nature de celle-ci.

Les propos qui suivent exposent d'abord un bref historique de l'implication des parties prenantes sous l'angle de lévolution de la pratique. Sont ensuite discutés les avantages et défis associés à cette implication. Il y est également question de la définition du concept de parties prenantes et de l'identification des approches évaluatives favorisant cette implication. La section conclut finalement en décrivant les différentes dimensions permettant de caractériser l'implication des parties prenantes et en ciblant les critères recensés dans la littérature afin de procéder à leur sélection.

\section{Perspective historique}

Une attention significative envers l'implication des parties prenantes apparait au courant des années 1990 et coïncide avec un changement de paradigme dans le développement de la pratique évaluative (Dubois, Champagne et Bilodeau, 2011), 
bien que certains évaluateurs, tels que Stake (1975), MacDonald (1976), Patton (1978) et Guba et Lincoln (1981), aient démontré un intérêt pour les approches inclusives avant cette période. En effet, une vision constructiviste de l'évaluation survient alors et gagne en popularité, délaissant l'ancienne approche positiviste réservée aux experts des méthodes scientifiques, au profit d'une évaluation plus ouverte et prenant assise au sein de modèles naturalistes et pluralistes (Dubois et coll., 2011; Fitzpatrick, Sanders et Worthen, 2011; Guba et Lincoln, 1989; Madaus, Stufflebeam et Scriven, 1989; Ridde et Dagenais, 2012).

La littérature évoque différentes raisons qui permettent d'expliquer la popularité plus marquée pour les approches impliquant les parties prenantes sur la fresque historique de la pratique évaluative, lesquelles constituent des avantages liés à cette implication et sont détaillées à la section suivante.

\section{Les avantages}

Les écrits témoignent de plusieurs avantages liés aux approches impliquant les parties prenantes. Parmi ceux-ci figure la prise en considération des principes déquité et de justice sociale (Cousins et Chouinard, 2012; House, 1993). En favorisant l'implication des parties prenantes, les évaluateurs cherchent ainsi à assurer leur représentation et participation dans le cadre du processus décisionnel relié à lévaluation, donnant la possibilité d'impliquer les acteurs clés concernés d'un programme lors de son évaluation, même si ces derniers sont associés à une clientèle difficilement joignable ou marginalisée (Hurteau, Houle et Marchand, 2012). Un autre bénéfice recensé concerne quant à lui l'utilisation de l'évaluation; en étant impliquées, les parties prenantes se sentent alors davantage représentées, favorisant une meilleure appropriation et compréhension de lévaluation et des résultats, maximisant du coup les potentialités d'utilisation (Cousins et Chouinard, 2012; Hofstetter et Alkin, 2003, Patton, 2008). Les parties prenantes demeurent par ailleurs une source première d'information relativement aux programmes évalués; leur implication au sein du processus évaluatif favorise ainsi lémergence de données de qualité et valides, lesquelles peuvent être également plus aisément triangulées (Ayers, 1987; Greene, 2000, Mark et coll., 2000; Nelson, 2009; O’Sullivan, 2012; Patton, 2002; Rodriguez-Campos, 2012). L’expérience de certains praticiens a permis de mettre en lumière le fait que cette implication obtient également un impact considérable sur le maintien de l'intérêt et de l'engagement des parties prenantes face aux programmes et à leur évaluation, sur l'accessibilité au terrain et sur le développement organisationnel (Beumier et Duquesne, 1988; Rodriguez-Campos, 2012; Tourneur et Rochez-Nimal, 1987). Il apparaît par ailleurs que la crédibilité accordée à une démarche dévaluation est tributaire de la contribution des parties prenantes (Hurteau, Houle, Marchand, Ndinga et coll., 2012).

\section{Les défis}

Si une majorité d'auteurs s'entend sur le fait que l'implication des parties prenantes est souhaitable, voire recommandée en raison des bénéfices qu'elle peut 
occasionner, cette même majorité partage également un consensus voulant que son opérationnalisation puisse être complexe et teintée de divers défis (Hurteau, 2009). Parmi ceux-ci, soulignons les enjeux inhérents à la protection de la crédibilité et de lobjectivité de la démarche et des résultats ainsi qu’à sa faisabilité, notamment en matière de temps et de disponibilité des ressources humaines et financières (Rodriguez-Campos, 2012). Des auteurs mentionnent par ailleurs la complexité liée au processus de sélection et de mobilisation des parties prenantes qui se révèle très souvent problématique (Hurteau, Houle et Marchand, 2012; Jacob et Ouvrard, 2009). Certains évaluateurs ont aussi relevé différentes difficultés associées à la qualité de l'implication, celle-ci étant entre autres tributaire de la clarification des rôles et responsabilités des acteurs impliqués, lesquels naffichent pas nécessairement de formation, d'habiletés ou de connaissances spécifiques en matière dévaluation (Cousins et Shulha, 2012; Rodriguez-Campos, 2012). Afin d’assurer la qualité de la démarche, lévaluateur doit aussi posséder de bonnes habiletés communicationnelles et démontrer d'excellentes aptitudes en matière de relations interpersonnelles, voire interculturelles, de façon à harmoniser les interactions entre et avec les parties prenantes, entre autres sur le plan de la gestion des conflits (Marchand, 2012). Il doit également être en mesure de faire preuve de flexibilité et de tolérance à légard des difficultés contextuelles pouvant émerger et relativement à la volonté des parties prenantes à s'impliquer dans la démarche (Garaway, 1995). Jacob et Ouvrard (2009) identifient des difficultés possibles attribuables au roulement de personnel parfois fréquent au sein de certains milieux, à l'existence de conflits pouvant mener à la réticence des parties prenantes à s'impliquer dans la démarche, à l’absence des acteurs clés et à la gestion de la diversité.

\section{Le concept de parties prenantes}

Le concept de parties prenantes fait globalement référence à l'ensemble des personnes interpellées ou affectées, à des degrés divers, par les résultats d’une évaluation (Chen, 2004; Fitzpatrick et coll., 2011; Mark et coll., 2006; Morra Imas et Rist, 2009; Orr, 2010; Patton, 2008), ou encore à l'ensemble des individus, groupes ou entités ayant des intérêts liés au programme ou à son évaluation (Brandon et Fukunaga, 2014; Théberge, 2011). Il est possible de retrouver différentes catégorisations au sein de la littérature du domaine de lévaluation afin de préciser le concept de parties prenantes, lesquelles affichent néanmoins des regroupements similaires (Greene, 2005; Daigneault et Jacob, 2012; Weiss, 1983). La typologie proposée par Daigneault et Jacob (2012) met en évidence le lien de proximité des groupes de parties prenantes par rapport aux programmes et demeure applicable dans la majorité des contextes évaluatifs. Cette typologie comporte quatre grands types de parties prenantes : 1 ) les décideurs, concepteurs et gestionnaires du programme; 2) les responsables de la mise en œuvre du programme; 3 ) les bénéficiaires directs et indirects ainsi que les tiers négativement affectés par un programme; et 4) la société civile et les citoyens. Le Tableau 1 expose une description de chacune de ces catégories et suggère divers exemples d’application. 
Tableau 1. Typologie de parties prenantes

\begin{tabular}{|c|c|c|}
\hline Types & Description & Exemples \\
\hline $\begin{array}{l}\text { Décideurs, } \\
\text { concepteurs et } \\
\text { gestionnaires }\end{array}$ & $\begin{array}{l}\text { Personnes politiquement, } \\
\text { légalement et/ou organisa- } \\
\text { tionnellement responsables } \\
\text { du programme et de son } \\
\text { évaluation }\end{array}$ & $\begin{array}{l}\text { Titulaire de charge pub- } \\
\text { lique (élu ou nommé), haut } \\
\text { fonctionnaire, gestionnaire } \\
\text { de mise en œuvre, président } \\
\text { et chef de direction d'un } \\
\text { organisme sans but lucratif, } \\
\text { etc. }\end{array}$ \\
\hline $\begin{array}{l}\text { Responsables de la } \\
\text { mise en œuvre }\end{array}$ & $\begin{array}{l}\text { Fonctionnaires et profes- } \\
\text { sionnels responsables de dis- } \\
\text { penser les biens et services } \\
\text { du programme aux groupes } \\
\text { cibles/bénéficiaires }\end{array}$ & $\begin{array}{l}\text { Fonctionnaire de première } \\
\text { ligne, infirmière, compt- } \\
\text { able, psychologue, agent de } \\
\text { développement internation- } \\
\text { al, travailleur social, enseig- } \\
\text { nant, agronome, etc. }\end{array}$ \\
\hline $\begin{array}{l}\text { Bénéficiaires directs } \\
\text { et indirects; tiers } \\
\text { négativement } \\
\text { affectés }\end{array}$ & $\begin{array}{l}\text { Personnes visées par le pro- } \\
\text { gramme pour modifier leur } \\
\text { comportement et/ou amé- } \\
\text { liorer leur bien-être (groupes } \\
\text { cibles et bénéficiaires); } \\
\text { autres personnes affectées } \\
\text { positivement ou négative- } \\
\text { ment par le programme }\end{array}$ & $\begin{array}{l}\text { Élève du niveau primaire, } \\
\text { parent, gais et lesbiennes, } \\
\text { famille immédiate d'un } \\
\text { délinquant juvénile, } \\
\text { conducteur en état } \\
\text { d'ébriété, habitant d'un } \\
\text { village nordique touché par } \\
\text { l'exploitation minière, etc. }\end{array}$ \\
\hline $\begin{array}{l}\text { Société civile et } \\
\text { citoyens }\end{array}$ & $\begin{array}{l}\text { Groupes organisés et citoy- } \\
\text { ens ayant un intérêt matériel, } \\
\text { politique et/ou scientifique } \\
\text { dans le programme et son } \\
\text { évaluation }\end{array}$ & $\begin{array}{l}\text { Groupes d'intérêts, syndicat, } \\
\text { groupe de réflexion, expert } \\
\text { indépendant, intellectuel, } \\
\text { comité, citoyen, etc. }\end{array}$ \\
\hline
\end{tabular}

Notes. Adapté de Conceptualiser et mesurer la participation à l'évaluation, par Daigneault et Jacob $(2012$, p. 242).

\section{Les approches}

La multiplicité des bénéfices associés à l'implication des parties prenantes dans le cadre de l'exercice évaluatif a permis de donner naissance à diverses approches évaluatives. Les écrits en dénombrent une variété, dont certaines se déclinent en sous-approches et/ou combinent des composantes de différentes approches. Il est également possible de retrouver une pluralité de propositions quant à la façon de classifier ces approches. Dans les travaux de Rodriguez-Campos (2012), trois grandes formes d'évaluation impliquant les parties prenantes sont identifiées : 1) lévaluation collaborative, où l'évaluateur contrôle le processus évaluatif tout en entretenant un engagement continuel avec les parties prenantes; 2) lévaluation participative, où le contrôle de lévaluation est partagé entre lévaluateur et les parties prenantes, lesquelles peuvent être amenées à planifier lévaluation, développer 
l'instrumentation nécessaire, collecter et analyser les données ou diffuser les résultats; et 3) lévaluation axée sur le renforcement des capacités, où les parties prenantes contrôlent et pilotent lévaluation, l'évaluateur jouant un rôle de soutien et d'accompagnement pour assurer la qualité et la rigueur du processus.

Par ailleurs, deux grandes orientations aux approches impliquant les parties prenantes se dégagent des écrits : 1) lévaluation avec visées pratiques (ou évaluation pratique) et 2) lévaluation avec visées transformatives (ou évaluation transformative) (Cousins et Chouinard, 2012; Cousins et Whitmore, 1998). L'évaluation avec visées pratiques se veut essentiellement pragmatique et se concentre sur l'utilisation des résultats, soutenant la prémisse que les résultats évaluatifs ont davantage de chances dêtre utilisés si les acteurs importants qui y sont associés ont été impliqués dans le processus qui a mené à leur production. Cette orientation regroupe entre autres l'evaluation axée sur les parties prenantes, l'évaluation axée sur l'utilisation et l'évaluation développementale (Cousins et Chouinard, 2012; Patton, 2008). Lévaluation transformative arbore quant à elle une fonction émancipatrice, qui favorise l'implication des parties prenantes afin d’engendrer un changement social, notamment en encourageant le pouvoir d'agir des parties prenantes (Cousins et Chouinard, 2012; Cousins et Whitmore, 1998). Lévaluation démocratique délibérative et lévaluation axée sur le renforcement des capacités figurent parmi les approches transformatives (Bélanger, 2011; Cousins et Chouinard, 2012; Fetterman, Kaftarian, et Wandersman, 1996; House et Howe, 2000).

\section{Les dimensions de l'implication}

L'implication des parties prenantes étant un concept vaste qui se concrétise dans la pratique sous différentes formes et variantes, comme discuté à la section précédente, des auteurs proposent de concevoir cette implication à partir de trois dimensions : 1) l'étendue de l'implication, 2) la diversité des participants impliqués et 3) le contrôle du processus évaluatif exercé par les parties prenantes (Cousins et Whitmore, 1998; Daigneault et Jacob, 2012). Ces dimensions, initialement introduites par Cousins et Whitmore (1998) puis retravaillées par Daigneault et Jacob (2012), demeurent essentielles à considérer lors de la mise en place d'une démarche impliquant les parties prenantes, de façon à bien circonscrire l'implication souhaitée des parties prenantes lors de la planification évaluative.

Létendue de l'implication fait référence au rôle joué par les parties prenantes relativement au sein des tâches techniques et opérationnelles de lévaluation. À quelles étapes évaluatives les parties prenantes seront-elles impliquées : lors de la conception de lévaluation; lors de la collecte des données; lors de la formulation des jugements et des recommandations; lors de la présentation et de la diffusion des résultats de lévaluation? La dimension de la diversité des participants impliqués, pour sa part, s'intéresse à la variété des groupes de parties prenantes en présence et peut être établie à partir d'une typologie ou d'une catégorisation de parties prenantes, telle que celle présentée dans le Tableau 1. Quels seront les groupes de parties prenantes impliqués dans le cadre de la démarche : les décideurs, concepteurs et gestionnaires du programme; les responsables de la mise en œuvre du programme; 
les bénéficiaires directs et indirects; la société civile? Finalement, le niveau de contrôle du processus évaluatif exercé par les parties prenantes est également à considérer afin de qualifier l'implication des parties prenantes. De quelle façon sera partagé ce contrôle entre l'évaluateur et les parties prenantes dans le déroulement de lévaluation? (Daigneault et Jacob, 2012)

\section{La sélection des parties prenantes}

De nombreux auteurs s'entendent pour affirmer que létape de sélection des parties prenantes demeure cruciale dans la bonne mise en œuvre d'une démarche impliquant les parties prenantes. Pourtant, la réalité en est souvent autrement, car le processus peut parfois échapper au contrôle des évaluateurs ou se voir amalgamé aux tâches régulières de l'évaluation (Hurteau, Houle et Marchand, 2012). Certains auteurs se sont tout de même penchés sur la question et proposent différents aspects à prendre à considération lorsque vient le temps de procéder à la sélection des parties prenantes (Bélanger, 2006; Bryk, 1983; Crosby et Bryson, 1992; House et Howe, 2000; Hurteau, Houle et Marchand, 2012; Mitchell, Agle et Wood, 1997).

Figurent d'abord les aspects individuels propres aux parties prenantes en présence. Parmi ceux-ci se retrouvent des caractéristiques sociodémographiques (âge, sexe, appartenance ethnique, langue parlée) qu'il importe de considérer afin de mettre en branle une démarche juste et équitable (Bamberger, 2000; Hurteau, Houle et Marchand, 2012). Vient ensuite s'ajouter l'importance des compétences relationnelles chez les parties prenantes sélectionnées (House et Howe, 2000; Hurteau, Houle et Marchand, 2012; King, 2005), de façon à ce que ces dernières puissent exercer un certain leadership en plus de faire preuve d'une éthique de travail, de posséder une capacité d'écoute et de respect et d’être aptes à communiquer habilement. La légitimité des parties prenantes demeure aussi une dimension essentielle. Il importe en ce sens de s'assurer que les revendications quelles expriment correspondent à des besoins réels pour l’amélioration du programme (House et Howe, 2000; Mathie et Greene, 1997; Mitchell et coll., 1997).

Des critères dordre relationnel sont également recensés dans la littérature afin de guider lévaluateur soucieux de sélectionner adéquatement les parties prenantes. Bien quelles puissent être difficiles à cerner sous tous les angles (Hurteau, Houle et Marchand, 2012), les dimensions de l'interaction entre l'intérêt et le pouvoir, le degré d'influence et le niveau de collaboration des parties prenantes au sein de l'organisation représentent des volets d'importance à bien étudier (Bélanger, 2006; Crosby et Bryson, 1992; Mitchell et coll., 1997). Il faut alors s'interroger, entre autres, sur la nature des avantages recherchés par les parties prenantes, leur pouvoir d'influence, leur légitimité au sein de l'organisation et l'historique de leur collaboration au sein de lorganisation (Bélanger, 2006; Crosby et Bryson, 1992; Mitchell et coll., 1997).

\section{DISCUSSION}

L'évaluation impliquant les parties prenantes s'inscrit dans un "nouveau » paradigme et aspire à être plus interactive et à laisser libre-cours aux négociations entre 
lévaluateur et les parties prenantes. Il s'agit là d'une démocratisation de la pratique en évaluation. Le rôle des parties prenantes au sein du processus évaluatif se module; alors que celles-ci endossaient une fonction plus passive essentiellement consultative sous le règne des approches antérieures, elles se voient dorénavant accorder davantage de pouvoir, pouvant être amenées à participer à la planification et/ou à la réalisation de lévaluation. Elles peuvent ainsi participer à la définition de lévaluation lors de la phase de planification, exprimant leurs attentes, préoccupations et besoins en informations. L'évaluateur est alors plus à même délaborer une démarche empreinte de sensibilité contextuelle, susceptible de revêtir une utilité et une crédibilité à leurs yeux, ce qui s'avère de haute importance pour l'utilisation de lévaluation et de ses résultats.

Comme démontré précédemment, les auteurs ont proposé des catégorisations de groupes de parties prenantes. Il serait toutefois erroné de croire que ces groupes sont homogènes; les intervenants d'un programme, qui relèvent du groupe « responsables de la mise en œuvre » selon la typologie de Daigneault et Jacob (2012), partageant très rarement la même opinion relativement à un programme. Ces regroupements ne sont par ailleurs pas exclusifs; à titre d'exemple, un enseignant à lécole primaire pouvant s'identifier spécifiquement à deux groupes de parties prenantes relativement à un programme d'aide aux devoirs, d'abord en lien avec ses fonctions professionnelles (responsables de la mise en œuvre), puis en lien avec sa vie personnelle et familiale en tant que père d'un enfant qui participe au programme (bénéficiaires directs et indirects). Les modélisations élaborées comprennent certainement leurs limites. Néanmoins, recourir à ce type de catégorisations de parties prenantes dans la pratique comprend une valeur ajoutée considérable. En effet, cela permet aux évaluateurs de prendre le temps de réfléchir à lensemble des personnes potentiellement affectées par un programme et par son évaluation, anticipant du coup les conséquences et impacts d'un tel exercice pour chacune d’elles. Par le fait même, les évaluateurs sont à mieux de percevoir si la démarche évaluative qu'ils pilotent parvient à rejoindre l'ensemble des groupes en présence, évitant doublier ou de négliger certains d'entre eux, ou à l'inverse, d’en survaloriser injustement.

Il demeure que le processus conduisant à la sélection des parties prenantes obtient une incidence majeure sur la qualité de la démarche dévaluation, étant directement lié à des enjeux déquité et de représentativité. Néanmoins, étant donné le caractère authentique et unique à chaque programme et à chaque situation évaluative, il s'avère plutôt complexe de dresser une liste de critères universels pour orienter adéquatement ce processus (Hurteau, Houle et Marchand, 2012). Il apparait tout à fait légitime de se questionner sur la pertinence de certains critères soulevés dans les écrits. À titre d’exemple, n’y a-t-il pas une contradiction évidente à sélectionner les parties prenantes sur la base de leurs compétences communicationnelles, évinçant de la sorte les individus ne possédant pas les compétences adéquates, alors que l'essence de la démarche prend assise sur un principe fondamental d'inclusion? Une réflexion quant aux critères discutés plus tôt permet toutefois d'indiquer les avenues à emprunter ou à éviter pour sélectionner judicieusement les parties prenantes. 
La littérature met en évidence différentes approches évaluatives, affichant leurs propres visées et variantes. Ces diverses formes d'approches ne sobservent cependant pas de façon aussi nette dans la pratique, les évaluateurs étant amenés à combiner les composantes de certains de ces types dévaluation en fonction du contexte dans lequel ils se retrouvent. Il n'est donc pas rare d'assister à la mise en place d'une démarche a priori axée sur la résolution de problèmes et entretenant des intentions pragmatiques, mais visant du même coup le renforcement des capacités des parties prenantes en présence. L'implication des parties prenantes se concrétise aussi dans la pratique sous différentes formes pouvant varier de presque nulle, à limitée, à substantielle puis à totale (Daigneault et Jacob, 2012). Du coup, il faut demeurer critique face à une démarche identifiée comme impliquant les parties prenantes, car la consultation, la participation et la collaboration ne se situent pas à la même position sur le « continuum de l'implication ». Ultimement, le choix de l'approche ou de la nature de l'implication souhaitée demeure déterminé selon le contexte du programme ainsi que les intentions sous-jacentes à la démarche. En ce sens, lévaluation impliquant les parties prenantes n'est pas toujours la panacée à toutes les situations, malgré ses nombreux bénéfices et avantages.

\section{CONCLUSION}

Les associations nord-américaines en évaluation ont intégré à leurs référentiels de compétences requises pour la pratique évaluative différentes orientations et considérations liées à la contribution des parties prenantes, preuve de l'enracinement du mouvement de démocratisation actuel (American Evaluation Association, 2004; Brandon et Fukunaga, 2014; Datta, 2006; Société canadienne dévaluation, 2010). Le présent article avait pour ambition de faire un tour d'horizon de la littérature en ce qui a trait à l'implication des parties prenantes. Les aspects développés ont permis de dresser un portrait synthétisé des diverses composantes inhérentes au concept d'implication des parties prenantes et sur lesquelles les évaluateurs ont le devoir de s'interroger avant de mettre en œuvre une évaluation qui favorise cette implication.

En somme, l'implication des parties prenantes apparaît comme une condition incontournable pour la pratique de plusieurs évaluateurs. Celle-ci comprend néanmoins de multiples défis et enjeux et nécessite à coup sûr le déploiement d'excellentes compétences chez les évaluateurs. Louvrage de Jacob et Ouvrard (2009) propose à cet égard d'intéressantes pistes d’actions pour remédier aux difficultés liées à l'implication des parties prenantes. Afin de bonifier la pratique et de parvenir à une meilleure compréhension des conditions gagnantes pour la mise en place d'une évaluation de qualité caractérisée par une implication des parties prenantes réussie, il est de mise de tirer profit des expériences passées et actuelles partagées par la communauté évaluative. La pratique gagnera certainement à s'intéresser aux perspectives des différents groupes de parties prenantes quant à leurs façons de recevoir l'arrivée et le déploiement d'une démarche dévaluation ainsi que de concevoir leurs rôles et responsabilités au sein 
d'un tel exercice. Pourrait-on mieux appréhender les attentes des parties prenantes et rendre lévaluation davantage crédible à leurs yeux en tenant compte de leurs perspectives à cet égard? L'auteure entend bien identifier des pistes de réponse à cette question dans le cadre de sa thèse.

\section{RÉFÉRENCES}

American Evaluation Association (2004). Guiding principles for evaluators. Récupéré le 9 avril $2018 \mathrm{de} \mathrm{http://www.eval.org/p/cm/ld/fid=51}$

Ayers, T.D. (1987). Stakeholders as partners in evaluation: A stakeholder-collaborative approach. Evaluation and Program Planning, 10(3), 263-271. https://doi.org/10.1016/ 0149-7189(87)90038-3

Bamberger, M. (2000). The evaluation of international development programs: A view from the front. American Journal of Evaluation, 21(1), 95-102. https://doi. org/10.1177/109821400002100108

Bélanger, J. (2006). Évaluation de la mise en œuvre d'interventions à déploiement variable : exemple d'utilisation pratique de la théorie. Mesure et évaluation en éducation, 29(3), 75-96.

Bélanger, J.-F. (2011). Lévaluation habilitative. Dans P.-M. Daigneault (éd.), Les approches théoriques en évaluation (p. 7-16). Québec: PERFEVAL.

Beumier, P. et Duquesne, F. (1988). Une approche méthodologique pour l'évaluation de la gestion des innovations pédagogiques : allers et retours entre la trame conceptuelle et le recueil des données. Mesure et évaluation en éducation, 11(2), 27-42.

Brandon, P.R. et Fukunaga, L.L. (2014). The state of empirical research literature on stakeholder involvement in program evaluation. American Journal of Evaluation, 35(1), 26-44. https://doi.org/10.1177/1098214013503699

Bryk, A.S. (1983). Editor's notes. Stakeholders-based evaluation. New Directions for Evaluation, 17, 1-2.

Chen, H.T. (2004). The roots and growth of theory-driven evaluation: An integrated perspective for assessing visibility, effectuality, and transferability. Dans M.C. Alkin (éd.), Evaluation roots: Tracing theorists' views and influences (pp. 113-129). Thousand Oaks, CA: Sage Publications.

Cousins, J.B. et Chouinard, J.A. (éds.). (2012). Participatory evaluation up close: An integration of research-based knowledge. Charlotte, NC: Information Age Publishing Inc.

Cousins, J.B. et Shulha, L.M. (2012). Létablissement des seuils de réussite dans le cadre d'une évaluation collaborative : une demarche complexe. Dans M. Hurteau, S. Houle et F. Guillemette (éds.), Lévaluation de programme axée sur le jugement crédible (pp. 137155). Québec: Presses de l'Université du Québec.

Cousins, J.B. et Whitmore, E. (1998). Framing participatory evaluation in understanding and practicing participatory evaluation. Dans E. Whitmore (éd.), New directions for evaluation (pp. 5-23). San Francisco: Jossey-Bass.

Crosby, B.C et Bryson, J.M. (1992). Leadership for the common good: Tackling public problems in a shared-power world. San Francisco: Jossey-Bass. 
Daigneault, P.-M. et Jacob, S. (2012). Conceptualiser et mesurer la participation à lévaluation. Dans V. Ridde et C. Dagenais (éds.), Approches et pratiques en évaluation de programmes (pp. 233-254). Montréal: Presses de l'Université de Montréal. https:// doi.org/10.4000/books.pum.5997.

Datta, L.E. (2006). The practice of evaluation challenges and new directions. Dans I.F. Shaw, J.C. Greene et M.M. Mark (éd.), The Sage handbook of evaluation (pp. 419-438). Thousand Oaks: Sage Publications. https://doi.org/10.4135/9781848608078.n19.

Dubois, C.-A., Champagne, F. et Bilodeau, H. (2011). Historique de l'évaluation. Dans A. Brousselle, F. Champagne, A.-P. Contandriopoulos et Z. Hartz (éds.), Lévaluation : concepts et methods (2 éd.) (pp. 27-48). Montréal: Presses de l'Université de Montréal. https://doi.org/10.4000/books.pum.6297.

Fetterman, D.M., Kaftarian, S.J. et Wandersman, A.H. (1996). Empowerment evaluation: Knowledge and tools for self-assessment and accountability. Thousand Oaks, CA: Sage Publications.

Fetterman, D.M., Rodriguez-Campos, L., Wandersman, A. et O’Sullivan, R.G. (2013). Collaborative, participative, and empowerment evaluation: Building a strong conceptual foundation for stakeholder involvement approaches to evaluation. American Journal of Evaluation, 35(1), 144-148. https://doi.org/10.1177/1098214013509875

Fitzpatrick, J.L., Sanders, J.R. et Worthen, B.R. (2011). Program evaluation. alternative approaches and practical guidelines ( $4^{\mathrm{e}}$ éd.). Boston: Pearson.

Garaway, G.B. (1995). Participatory evaluation. Studies in Educational Evaluation, 21(1), 85-102. https://doi.org/10.1016/0191-491X(95)00007-H

Greene, J.C. (2005). Stakeholder. Dans S. Mathison (éd.), Encyclopedia of evaluation (p. 397). Thousand Oaks, CA: Sage Publications.

Greene, J.C. (2000). Challenges in practicing deliberative democratic evaluation [Special issue: Evaluation as a democratic process: promoting inclusion, dialogue, and deliberation]. New Directions for Evaluation, 85, 13-26. https://doi.org/10.1002/ev.1158.

Guba, E.G. et Lincoln, Y.S. (1981). Effective evaluation: Improving the usefulness of evaluation results through responsive and naturalistic approaches. New Jersey: John Wiley \& Sons.

Guba, E.G. et Lincoln, Y.S. (1989). Fourth generation evaluation. Newbury Park: Sage Publications.

Hofstetter, C. et Alkin, M.C. (2003). Evaluation use revisited. Dans T. Kellaghan et D. L. Stufflebeam (éds.), International handbook of educational evaluation (pp. 197-222). Boston: Kluwer Academic Publishers.

House, E.R. (1993). Professional evaluation: Social impact and political consequences. Thousand Oaks, CA: Sage Publications.

House, E.R. et Howe, K.R. (2000). Deliberative democratic evaluation [Special issue: Evaluation as a democratic process: promoting inclusion, dialogue, and deliberation]. New Directions for Evaluation, 85, 3-12. https://doi.org/10.1002/ev.1157

Hurteau, M. (2009). Évaluation des programmes : ses visées? qui la pilote? et qui y participe? Dans L. Mottier Lopez et M. Crahay (éds.), Évaluations en tension : entre la régulation des apprentissages et le pilotage des systèmes (pp. 113-126), Neuville La Louve: De Boeck. 
Hurteau, M., Houle, S. et Marchand, M.-P. (2012). La sélection des parties prenantes : un enjeu important parce que toujours problématique. Dans M. Hurteau, S. Houle et F. Guillemette (éds.), Lévaluation de programme axée sur le jugement crédible (pp. 115136). Québec: Presses de l’Université du Québec.

Hurteau, M., Houle, S., Marchand, M.-P., Ndinga, P., Guillemette, F. et Schleifer, M. (2012). Les processus de production et de crédibilisation du jugement en évaluation. Dans M. Hurteau, S. Houle et F. Guillemette (éds.), Lévaluation de programme axée sur le jugement credible (pp. 77-100). Québec: Presses de l'Université du Québec.

Jacob, S. et Ouvrard, L. (2009). Lévaluation participative. Avantages et difficultés d'une pratique innovante. Cahier performance et evaluation, $\mathrm{n}^{\circ} 1$.

King, J.A. (2005). Participatory evaluation. Dans S. Mathison (éd.), Encyclopedia of evaluation (pp. 291-294). Thousand Oaks, CA: Sage Publications. https://doi. org/10.4135/9781412950558.n398.

MacDonald, B. (1976). Evaluation and the control of education. Dans D. Tawney (éd.), Curriculum evaluation today: Trends and implications (pp. 125-134). Schools Council Research Studies Series. London: Macmillan.

Madaus, G., Stufflebeam, D. et Scriven, M. (1989). Program evaluation: A historical overview. Dans G. Madaus, D. Stufflebeam et M. Scriven (éds.), Evaluation models (pp. 3-22). Boston: Kluwer-Nijhoff.

Marchand, M.-P. (2012). La contribution de l'évaluateur aux processus de production et de crédibilisation du jugement. Dans M. Hurteau, S. Houle et F. Guillemette (éds.), Lévaluation de programme axée sur le jugement credible (pp. 157-174). Québec: Presses de l’Université du Québec.

Mark, M.M., Greene, J.C. et Shaw, I.F. (2006). Introduction : the evaluation of policies, programs and practices. Dans I.F. Shaw, J.C. Greene et M.M. Mark (éds.), The Sage handbook of evaluation (pp. 1-30). Thousand Oaks. CA: Sage Publications. https:// doi.org/10.1016/j.cossms.2007.08.003.

Mark, M.M., Henry, G.T. et Julnes, G. (2000). Evaluation: An integrated framework for understanding, guiding, and improving policies and programs. San Francisco: JosseyBass.

Mathie, A. et Greene, J.C. (1997). Stakeholder participation in evaluation: How important is diversity? Evaluation and Program Planning, 20(3), 279-285. https://doi.org/10.1016/ S0149-7189(97)00006-2

Mitchell, R.K., Agle, B.R. et Wood, D.J. (1997). Toward a theory of stakeholder identification and salience: Defining the principle of who and what really counts. Academy of Management Review, 22(4), 853-886.

Morra Imas, L.G. et Rist, R.C. (2009). The road to results, designing and conducting effective development evaluations. Washington: The International Bank for Reconstruction and Development/The World Blank. https://doi.org/10.1596/978-0-8213-7891-5.

Nelson, R.K. (2009). An exploration of the factors that infuence participant selection in participatory evaluation. Thèse de doctorat, University of Minnesota.

O'Sullivan, R.G. (2012). Collaborative evaluation within a framework of stakeholderoriented evaluation approaches. Evaluation and Program Planning, 35(4), 518-522. https://doi.org/10.1016/j.evalprogplan.2011.12.005 
Orr, S.K. (2010). Exploring stakeholders values and interests in evaluation. American Journal of Evaluation, 31(4), 557-569. https://doi.org/10.1177/1098214010372668

Patton, M.Q. (1978). Utilization-focused evaluation. Thousand Oaks, CA: Sage.

Patton, M.Q. (2002). Qualitative research and evaluation methods (3e éd.). Thousand Oaks, CA: Sage Publications.

Patton, M.Q. (2008). Utilization-focused evaluation (4e éd.). Thousand Oaks, CA: Sage Publications.

Ridde, V. et Dagenais, C. (2012). Introduction générale à l'évaluation de programmes. Dans V. Ridde et C. Dagenais (éds.), Approches et pratiques en évaluation de programmes (pp. 13-32). Montréal: Presses de l'Université de Montréal. https://doi.org/10.4000/ books.pum. 5968 .

Rodriguez-Campos, L. (2012). Stakeholder involvement in evaluation: Three decades of the American journal of evaluation. Journal of MultiDisciplinary Evaluation, 8(17), 57-79.

Schwandt, T.A. (2009). Toward a practical theory of evidence for evaluation. Dans S.I. Donaldson, C.A. Christie et M.M. Mark (éds.), What counts as credible evidence in applied research and evaluation practice? (pp. 197-212). Los Angeles: Sage Publications. https://doi.org/10.4135/9781412995634.d18.

Société canadienne d'évaluation (2010). L'évaluation au Canada : référentiel des compétences professionnelles requises à son exercice. Récupéré le $1^{\mathrm{er}}$ septembre 2011 de http://www. evaluationcanada.ca/txt/2_competences_cdn_pratique_evaluation.pdf.

Stake, R.E. (1975). Evaluating the arts in education: A responsive approach. Columbus: Merrill.

Théberge, F. (2011). Lévaluation démocratique délibérative. Dans P.-M. Daigneault (éd.), Les approches théoriques en evaluation (pp. 23-30). Québec: PERFEVAL.

Tourneur, Y. et Rochez-Nimal, P. (1987). Macro-évaluation : finalités, objets détude et méthodes. Mesure et Évaluation en Éducation, 10(3), 29-60.

Weiss, C.H. (1983). Toward future of stakeholder approaches in evaluation [Stakeholderbased education]. New Directions for Evaluation, 17, 83-96. https://doi.org/10.1002/ ev.1328.

\section{PRÉSENTATION D'AUTEUR}

Marie-Pier Marchand est étudiante au doctorat en éducation à l'Université du Québec à Montréal. Dans le cadre de sa thèse, elle s'intéresse à la contribution des différents groupes de parties prenantes au sein des démarches d'évaluation. Elle s'est également impliquée dans la communauté évaluative afin de soutenir le développement et l'insertion des évaluateurs émergents et agit maintenant à titre de chargée de cours et d'assistante de recherche. 